\title{
Streamlines in stationary homogeneous isotropic turbulence and fractal-generated turbulence
}

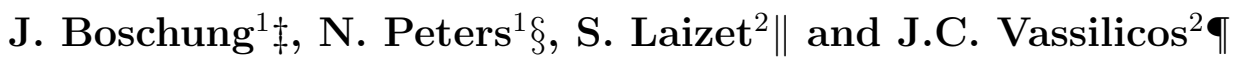 \\ ${ }^{1}$ Institute for Combustion Technology, RWTH Aachen University, Templergraben 64, \\ 52062 Aachen, Germany \\ ${ }^{2}$ Turbulence, Mixing and Flow Control Group, Department of Aeronautics, Imperial \\ College London, United Kingdom
}

E-mail: j.boschung@itv.rwth-aachen.de

\begin{abstract}
We compare streamline statistics in stationary homogeneous isotropic turbulence and in turbulence generated by a fractal square grid. We examine streamline segments characterised by the velocity difference $\Delta u$ and the distance $l$ between extremum points. We find close agreement between the stationary homogeneous isotropic turbulence and the decay region of the fractal-generated turbulence as well as the production region of the fractal flow for small segments. The statistics of larger segments are very similar for the isotropic turbulence and the decay region, but differ for the production region. Specifically, we examine the first, second and third conditional mean $\left\langle[\Delta u]^{n} \mid l\right\rangle$. Noticeably, non-vanishing $\left\langle[\Delta u]^{n} \mid l\right\rangle$ for $n=1,3$ are due to an asymmetry of positive and negative segments, i.e. those for which $\Delta u>0$ and $\Delta u<0$, respectively. This asymmetry is not only kinematic, but also due to dissipative effects and therefore $\left\langle[\Delta u]^{n} \mid l\right\rangle$ contain cascade information.
\end{abstract}

Keywords: Fluid dynamics, Turbulence, Streamlines

$\ddagger$ Corresponding author: j.boschung@itv.rwth-aachen.de

$\S$ n.peters@itv.rwth-aachen.de

|| s.laizet@imperial.ac.uk

I j.c.vassilicos@imperial.ac.uk 


\section{Introduction}

It has been known since Simmons \& Salter (1934) that grid-generated turbulence consists of a production region at the immediate vicinity of the grid where the turbulence increases with streamwise distance from the grid and a decay region downstream of this production region where the turbulence decays with streamwise distance from the grid. The grids used by Simmons \& Salter (1934) were regular. Hurst \& Vassilicos (2007) confirmed their finding for space-filling fractal square grids but also showed that for these grids the production region is significantly extended in size and the turbulence intensity in it is significantly reduced. This dual property of space-filling fractal square grids made the production region much easier to access experimentally than with regular grids. Gomes-Fernandes, Ganapathisubramani \& Vassilicos (2015) and Laizet, Nedić \& Vassilicos (2015a) used a space-filling fractal square grid to study the production region with Particle Image Velocimetry (PIV) and Hot Wire Anemometry respectively and demonstrated the presence in this region of a very well-defined $-5 / 3$ power law shape in the turbulence energy spectrum even though the turbulence in this region is nonhomogeneous, non-isotropic and non-gaussian as it consists of both potential and vortical flow. The turbulence in the decay region is well mixed and the fluctuating velocities there are gaussian and also much more homogeneous and isotropic. The turbulence in the decay region is also out of Richardson-Kolmogorov equilibrium and a new, nonequilibrium, scaling for the turbulence dissipation holds in a significant streamwise extent of this region (see review by Vassilicos (2015)). Goto \& Vassilicos (2015) have shown that this non-equilibrium scaling is also present in Direct Numerical Simulations (DNS) of periodic unsteady turbulence (decaying turbulence being an example of such turbulence) both for the dissipation rate and the interscale energy flux in an intermediate range of wavenumbers.

Streamline segments and their statistics have recently been used by Schaefer, Gampert \& Peters (2012a) and Schaefer, Gampert \& Peters (2013) as a way to characterise small-scale geometry of turbulence fluctuations. They analysed a variety of turbulent flows and found the same streamline segment statistics in all the flows they interrogated. However they did not apply their streamline segment analysis to turbulent flows with non-equilibrium dissipation scaling and to the production region of a gridgenerated turbulence. The PIV results on the generalised Kàrmàn-Howarth equation obtained by Gomes-Fernandes et al. (2015) suggest that there is a combined forward

and inverse cascade in different directions in the production region. It is therefore of interest to test the universality of streamline segment statistics in the production region and the non-equilibrium decay region of grid-generated turbulence. In this paper we compare streamline segment statistics obtained in a DNS of statistically stationary periodic turbulence with streamline segment statistics obtained in the production and the decay regions of a DNS of fractal-generated turbulence. The DNS of fractalgenerated turbulence that we use here is that of Laizet et al. (2015a). 


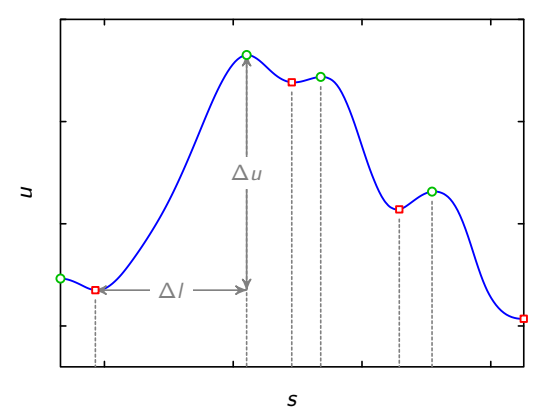

Figure 1. Projection of the velocity field $u_{i}$ onto a streamline. Streamline segments are defined by the local minima $\square$ and maxima $\bigcirc$ of the projected velocity $u=t_{i} u_{i}$ and characterised by their curvilinear length $l$ along the streamline coordinate $s$ and velocity difference $\Delta u$.

\section{Streamline segments}

Streamlines in a frozen flow field are lines tangent to the vector $t_{i}=u_{i} / u$ where $u=\sqrt{u_{i}^{2}}$ is the absolute value of the velocity. The curvilinear coordinate along the arc length of the streamline is denoted by $s$ in this paper. Streamlines are in principle infinitely long, unless they hit a stagnation point where all three velocity components vanish. This is why Wang (2010) has proposed to split streamlines into streamline segments which extend between local extreme points of $u$ along the streamline. Streamline segments are bound by two extrema, i.e. points where the velocity derivative with respect to the arc length $s$ vanishes. He further characterized streamline segments as positive or negative, depending on the sign of the gradient: within positive segments $\partial u / \partial s>0$; within negative segments $\partial u / \partial s<0$. Thus the flow along positive segments is accelerated, while it is decelerated along negative segments. Streamline segments are therefore characterised by the velocity difference $\Delta u$ between two local extrema and the arc distance $l$ between them, see figure 1 , where $\Delta u>0$ for positive segments and $\Delta u<0$ for negative segments.

In this paper, we choose the fluctuating velocity field for our analysis instead of the absolute velocity to make the statistics Galilean-invariant. For the statistically stationary periodic turbulence dataset, $\left\langle u_{i}\right\rangle=0$ by definition. For the fractal grid, this is not the case, therefore the mean value has been subtracted. We start a streamline at every (equally spaced) grid point. The streamline is then traced by following its unit tangent vector both in positive and negative direction using a Runge-Kutta scheme to adapt the step-size where the step-size is decreased when the velocity is very low. We use a tricubic interpolation scheme based on a third-degree spline to interpolate the velocity field between grid points. The tracing is stopped when the boundary of the domain is reached. As streamlines are not invariant with respect to time, we keep the velocity field frozen and we study streamline segment statistics in specific realisations of the turbulence obtained for specific times. 

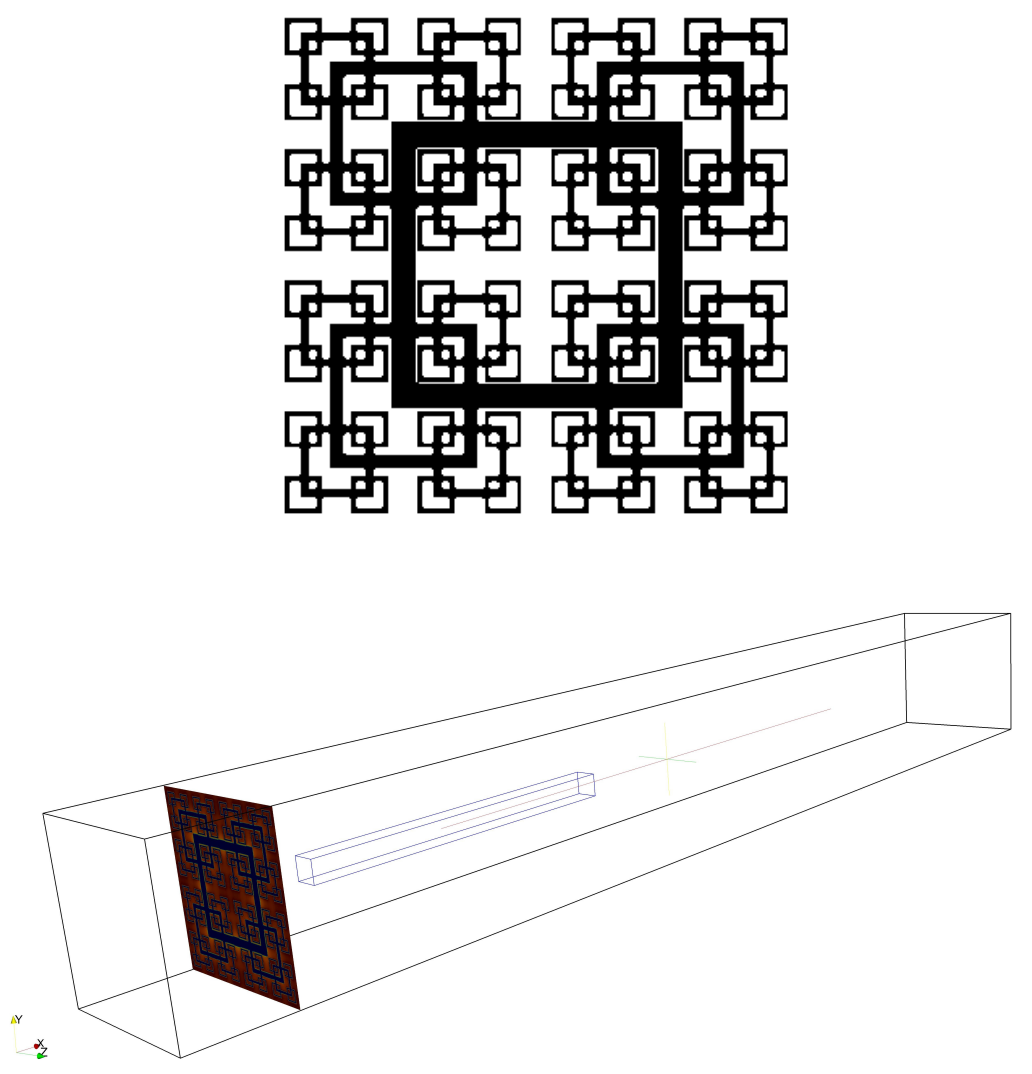

Figure 2. Diagram of the fractal square grid used in this study and illustration of the computational domain where the subdomain used for this study is highlighted in blue.

\section{Dataset description}

\subsection{Fractal DNS}

3.1.1. Description of the grid As shown in figure 2, we consider a fractal square grid with a square pattern formed by 4 bars (see Hurst \& Vassilicos (2007) for a detailed description of fractal square grids). It is based on 4 fractal iterations (with $4^{j}$ patterns at iteration $j$ ) and the ratio $t_{r} \equiv t_{\max } / t_{\min }$ between the lateral thickness $t_{\max }$ of the bars making the largest pattern and the lateral thickness $t_{\min }$ of the smallest one is equal to 8.5. $L_{j}$ with $j=0,1,2,3$ represents the length of the bars for each fractal iteration. The blockage ratio $\sigma$ of our turbulence-generating grid is defined as the ratio of its total area in the transverse plane to the area $T^{2}=L_{y} \times L_{z}$ and is equal to $41 \%$. Unlike regular grids, multiscale/fractal grids do not have a well-defined mesh size. This is why Hurst \& Vassilicos (2007) introduced an effective mesh size for multiscale grids, $M_{\text {eff }}=4 T^{2} \sqrt{1-\sigma} / L_{T G}$ where $L_{T G}$ is the total perimeter length in the $(y-z)$ plane of the fractal grid. Here, we have $M_{\text {eff }}=8.7 t_{\text {min }}$. Note finally that the streamwise thickness of the bars is $3.2 t_{\min }$. 
3.1.2. Numerical Methods The incompressible Navier-Stokes equations are solved using the high-order flow solver Incompact3d, adapted to parallel supercomputers thanks to a highly scalable 2D domain decomposition library and a distributed FFT interface (Laizet \& Li 2011). Sixth-order compact finite-difference schemes are used for the spatial differentiation whereas an explicit third-order Adams-Bashforth scheme is used for the time integration. To treat the incompressibility condition, a fractional step method requires solving a Poisson equation. This equation is fully solved in spectral space, via the use of relevant 3D Fast Fourier Transforms combined with the concept of modified wave number (Lele 1992). Note that the pressure mesh is staggered from the velocity one by half a mesh, to avoid spurious pressure oscillations. The divergence-free condition is ensured up to machine accuracy. The modeling of the fractal grid is performed using an Immersed Boundary Method (IBM) based on a direct forcing approach that ensures the no-slip boundary condition at the grid walls. The idea is to force the velocity to zero at the wall of the grid, as our particular Cartesian mesh does conform with the geometries of the grid. It mimics the effects of a solid surface on the fluid with an extra forcing in the Navier-Stokes equations. More details about the present code and its validation, especially the original treatment of the pressure in spectral space, can be found in Laizet \& Lamballais (2009).

3.1.3. Numerical set-up The computational domain in the streamwise $x$ and the two lateral $y$ and $z$ directions is $L_{x} \times L_{y} \times L_{z}=16 L_{0} \times 2 L_{0} \times 2 L_{0}$ discretized on a Cartesian mesh using $n_{y} \times n_{z}=720 \times 720$ mesh nodes in lateral planes and $n_{x}=5761$ in the streamwise direction. This high resolution was recommended by Laizet et al. (2015a) especially for the production region. The coordinate system's origin is placed at the centre of the grid which is located at a distance of $1.25 L_{0}$ from the inlet of the computational domain in order to avoid spurious interactions between the grid and the inlet condition. We assume a fluid of uniform density and kinematic viscosity $\nu$. Inflow/outflow boundary conditions are used in the streamwise direction and periodic boundary conditions are used in the two lateral directions. The inflow and initial conditions for the velocity field are $\mathbf{u} \equiv\left(u_{1}, u_{2}, u_{3}\right)=\left(U_{\infty}, 0,0\right)$ where $U_{\infty}$ is a constant streamwise velocity $\left(u_{1}\right.$ is the streamwise velocity component and $\left(u_{2}, u_{3}\right)$ are the two lateral velocity components corresponding to $(y, z))$. The outflow condition is a standard $1 \mathrm{D}$ convection equation.

For this particular study, data are collected for 5 time-independent snapshots of a $3 \mathrm{D}$ subdomain (see figure 2 ) of size $4.25 L_{0} \times 0.28 L_{0} \times 0.28 L_{0}$ with $1530 \times 101 \times 101$ mesh nodes. The $3 \mathrm{D}$ subdomain is centred around the centreline of the grid starting from a distance $0.15 x_{*}$ downstream of the grid and extending to a distance $0.55 x_{*}$, where $x_{*}$ is the wake interaction length scale introduced by Mazellier \& Vassilicos (2010) and which is equal to $L_{0}^{2} / t_{0}$. The fractal flow data used in Figures 4 to 6 are taken from the production and the decay regions explicitly shown in Figure 3. For this fractal square grid simulation, the values of the Taylor-based Reynolds number $R e_{\lambda}=u_{r m s} \lambda / \nu$, the Taylor microscale $\lambda=\sqrt{u_{r m s} /(\partial u / \partial x)^{2}}$ and the integral scale $L$ are varying with 


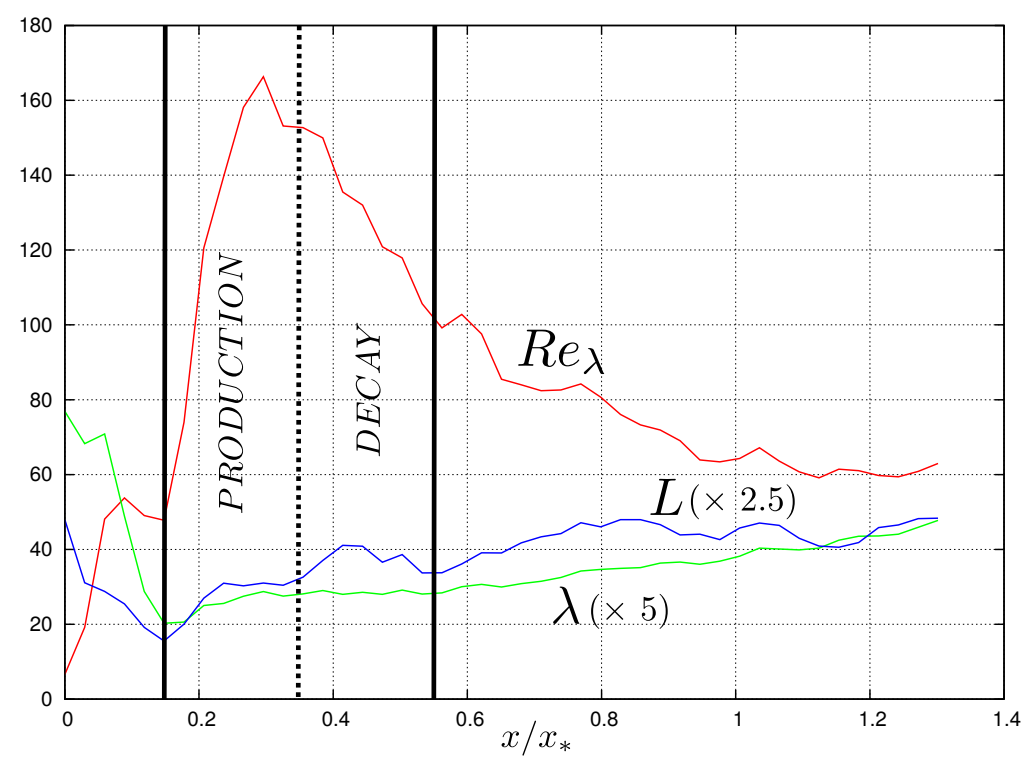

Figure 3. Streamwise evolution along the centreline for the fractal square grid of the Taylor-based Reynolds number $R e_{\lambda}$, the Taylor microscale $\lambda$ and the integral scale $L$. The production region (from $x=0.15 x_{*}$ to $x=0.35 x_{*}$ ) and the decay region (from $x=0.15 x_{*}$ to $x=0.35 x_{*}$ ) where the data are collected is highlighted around the $R e_{\lambda}$ peak.

streamwise distance from the grid and their values as functions of $x / x_{*}$ can be seen in Figure 3 .

\subsection{Isotropic DNS}

We compare the fractal grid data to statistically stationary periodic turbulence at a similar Taylor-based Reynolds number $R e_{\lambda}=184$ with $R e_{\lambda}=u_{r m s} \lambda / \nu$ where $\lambda=\sqrt{10 \nu\langle k\rangle /\langle\varepsilon\rangle}=0.166$ denotes the Taylor scale and $\langle k\rangle=\left\langle u_{i} u_{i}\right\rangle / 2=11.42$ the mean kinetic energy. Furthermore $\langle\varepsilon\rangle=2 \nu\left\langle\left(\partial u_{i} / \partial x_{j}+\partial u_{j} / \partial x_{i}\right)^{2}\right\rangle=10.30$ denotes the mean energy dissipation, where $\nu=0.0025$ is the kinematic viscosity. The dataset has been calculated on the JUQUEEN supercomputer at Forschungszentrum Jülich using a pseudo-spectral code with MPI/OpenMP parallelisation. The three-dimensional Navier-Stokes equations were solved in rotational form, where all terms but the nonlinear term were evaluated in spectral space. For a faster computation, the non-linear term is evaluated in physical space. The computational domain is a box with periodic boundary conditions and length $2 \pi$. For de-aliasing, the scheme of Hou \& Li (2007) has been used. For the temporal advancement, a second order Adams-Bashforth scheme is used in case of the non-linear term, while the linear terms are updated using a CrankNicolson scheme. To keep the simulation statistically steady, the stochastic forcing scheme of Eswaran \& Pope (1988) is applied. The 2DECOMP\&FFT library ((Li \& 
Laizet 2010)) has been used for spatial decomposition and to perform the fast Fourier transforms. The dataset was computed on a computational mesh with $1024^{3}$ grid points. $\eta=\left(\nu^{3} /\langle\varepsilon\rangle\right)^{1 / 4}=0.0062$ is the Kolmogorov length scale with corresponding time scale $\tau_{\eta}=(\nu /\langle\varepsilon\rangle)^{1 / 2}=0.016 . L=0.97$ is the integral length scale, here computed using the energy spectrum and $\tau=\langle k\rangle /\langle\varepsilon\rangle=1.11$ is an estimate of the integral time scale. The integral length scale $L$ is small compared to the size $2 \pi$ of the boxes in order to reduce the influence of the periodic boundary condition. Our data is well resolved with $k_{\max } \eta \geq 2.66$. We specifically chose a dataset with a Reynolds number similar to the grid data described above. We also briefly look at the joint statistics of $\Delta u$ and $l$ for an artificial velocity field with vanishing velocity derivative skewness. This velocity field was obtained by randomising the phases of the Fourier-transformed velocity components of the isotropic data set while keeping their amplitudes fixed. Continuity is then retained by projecting the Fourier-transformed velocity in the plane normal to the wave vector. The skewness of the longitudinal velocity gradient is then decreased to -0.00295 as compared to -0.54 for the original isotropic velocity field.

\section{Results}

\subsection{Joint $p d f$}

Figure 4 shows the joint probability density function (jpdf) $P(\Delta u, l)$ for statistically stationary periodic turbulence (figure 4(a)), the fractal flow in the production region close to the grid (figure 4(b) and in the near-field decay region further downstream (figure 4(c) normalised by their respective mean segment length $l_{m}$ and standard deviation $\sigma=\left\langle[\Delta u]^{2}\right\rangle^{1 / 2}$.

In the case of statistically stationary periodic turbulence (fig. 4(a)p, we find the same shape as Wang (2010), namely a noticeable asymmetry. Specifically, positive segments are longer on average than negative segments. This is consistent with their positive velocity difference $\Delta u>0$ which stretches positive segments, while negative segments are compressed by their negative velocity difference $\Delta u<0$. It follows that the absolute

mean velocity difference of positive segments $\langle|\Delta u| \mid \Delta u>0\rangle$ is necessarily smaller than that of negative segments, as positive and negative segments along a streamline alternate and the velocity $u$ is finite. This is confirmed by the shape of the jpdf and in agreement with the negative skewness of the velocity derivative (Wang 2010). As $|\Delta u|>0$, both wings of the jpdf should be separated. This is not the case for the jpdfs in figure 4 due to finite binning. A model for the jpdf has been given by Schaefer et al. (2013), which shows good qualitative agreement with data from DNS.

The jpdf for the production region close to the fractal grid looks qualitatively similar, albeit more symmetrical. Further downstream, in the near-field decay region where the turbulence is out of Richardson-Kolmogorov equilibrium, the jpdf (fig. 4) approaches that of the statistically stationary periodic turbulence which is in equilibrium by virtue of the near-instantaneous balance between dissipation and power input 


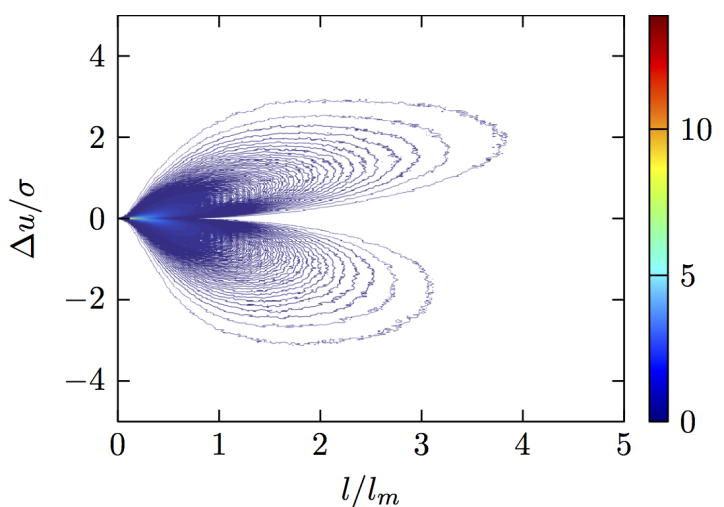

(a)

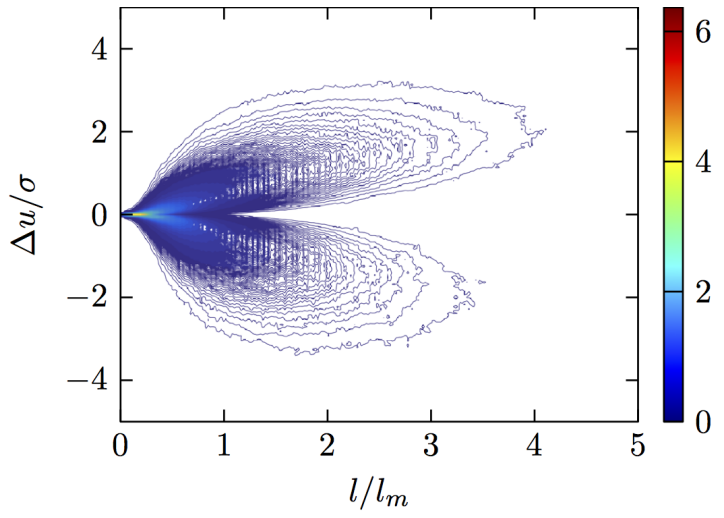

(c)

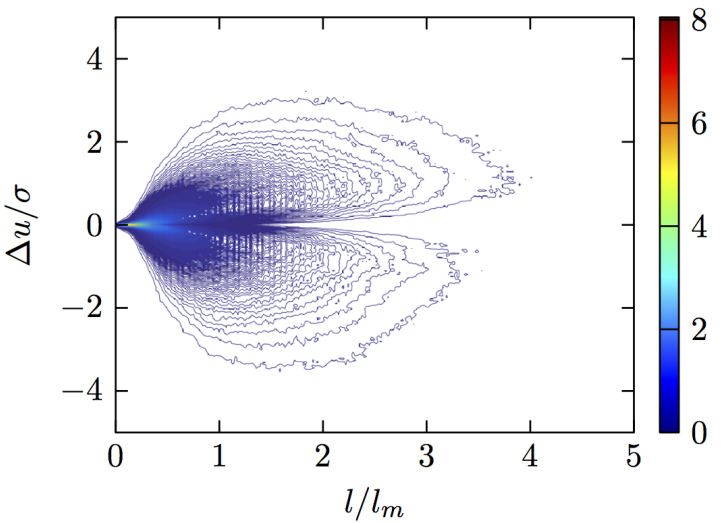

(b)

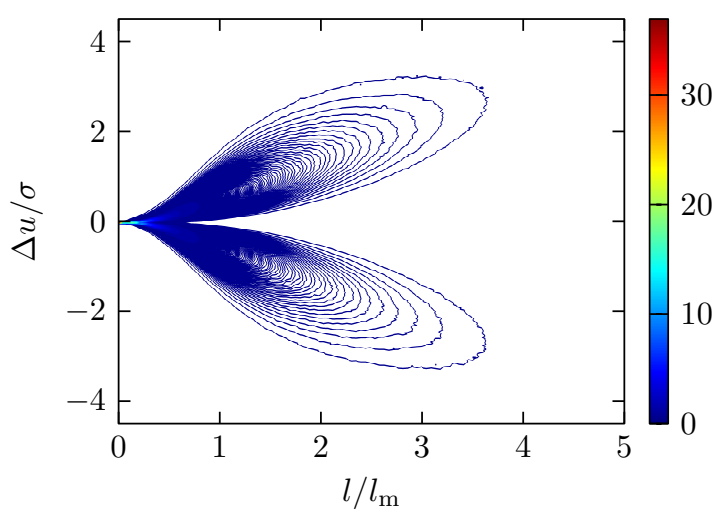

(d)

Figure 4. Joint PDF $P(\Delta u, l)$. (a) Periodic dataset, (b) production region, (c) decay region, (d) vanishing skewness.

required to keep it statistically stationary.

Finally, we show the jpdf of the artificial, vanishing velocity derivative skewness data in figure 4(d), The shape of the jpdf is symmetric, i.e. positive and negative segments have the same statistics. As the energy cascade causes the velocity derivative skewness to be non-zero (and negative), this result suggests that the asymmetry of the jpdf in (fig. 4)a,b,c is a reflection of the energy cascade at length-scales which are multiples/fractions but of the order of the mean segment length $l_{m}$. This is not a trivial result, in particular because $l_{m}$ is a dissipative-range length-scale since $l_{m}=\sqrt{\eta \lambda}$, where $\eta$ is the Kolmogorov length-scale and $\lambda$ is the Taylor length-scale, see Schaefer, Gampert \& Peters (2012b).

\subsection{Marginal pdfs}

The marginal probability density functions (pdfs) of $l$ and $\Delta u$ normalised by $l_{m}$ and $\sigma$ are shown in figure 5 both plotted linearly and semi-logarithmically. The vanishing velocity skewness data has been used to make the connection between turbulence cascade 


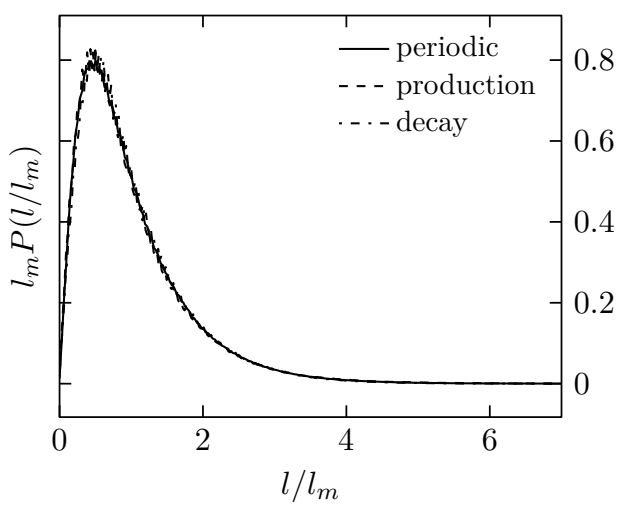

(a)

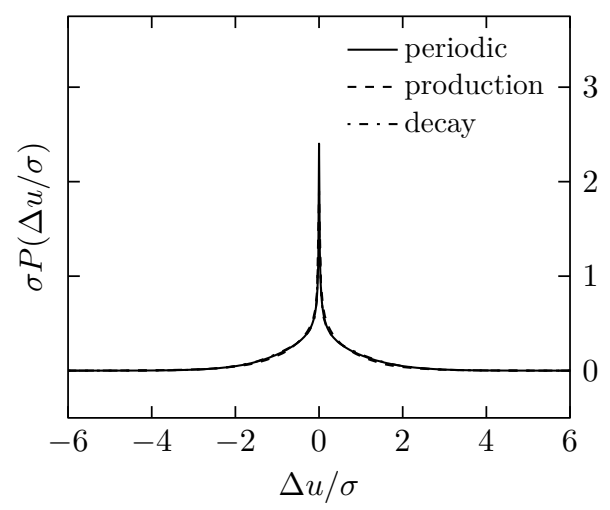

(c)

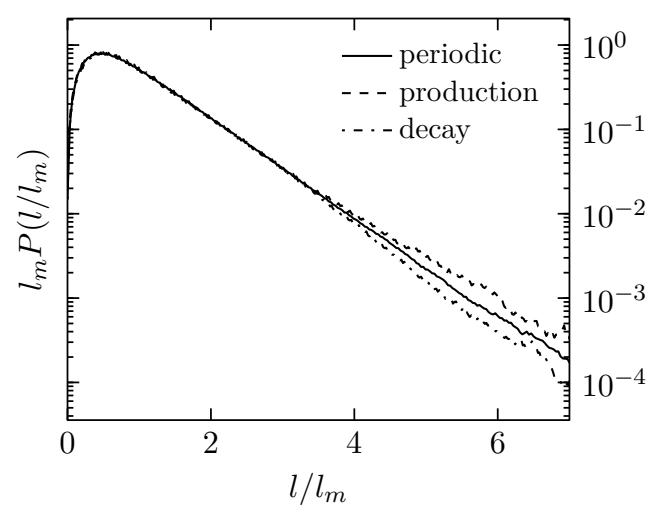

(b)

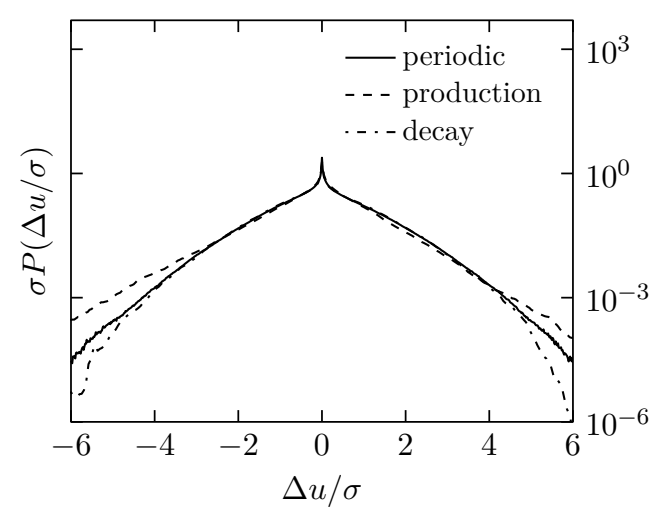

(d)

Figure 5. Normalised marginal PDFs. (a) and (b) $P(l)$, (c) and (d) $P(\Delta u)$.

and the streamline segment statistics and is not needed any longer. Is is therefore not included in the figures and discussion of this and next sub-sections.

Noticeably, the normalised pdf of the segment length collapses for the three cases of homogeneous isotropic turbulence and the production and decay region of the fractal flow. The pdfs peak at $l / l_{m} \sim 0.6$ and show a linear behaviour for $l / l_{m} \rightarrow 0$ and an exponential tail for $l / l_{m} \rightarrow \infty$. This exponential tail is especially highlighted in figure $5(\mathrm{~b})$ and corresponds to a random cutting-/reconnection process acting on large segments (Schaefer et al. 2012a). Small segments $l / l_{m} \rightarrow 0$ are dominated by a drift towards smaller $l$ due to molecular diffusion, in agreement with the linear rise observed in figure 5(a) (Schaefer et al. 2012a). Schaefer et al. (2012a) derived a model for the pdf of $l$, which agrees very well with DNS data. Noticeably, their model includes a small Reynolds number dependency of the cutting-/reconnection process. Note that there are not that many segments with very high $l$ and that their number is even less in the case of the fractal dataset. We can therefore not rule out that the tails may not be converged and that they would collapse if we had more data. Hence, we can not conclude whether the production and the non-equilibrium decay regions have same or different marginal distributions for segment lengths $l$ larger than $3 l_{m}$ when normalised with $l_{m}$. 
The marginal pdf of the velocity difference $\Delta u$ at the end points of the segments normalised by $\sigma=\langle\Delta u\rangle^{1 / 2}$ is shown in figure 5(c) and $5(\mathrm{~d})$. We find that the normalised $P(\Delta u)$ significantly deviate from a normal distribution and that they collapse for small velocity differences $\Delta u$. The tails of the pdfs however do not collapse; especially negative segments differ. We find the production region pdf to be more symmetric than in both the statistically stationary periodic turbulence and the near-field decaying turbulence, in agreement with their joint pdfs in figure 4. It should be mentioned that the nonnormalised pdfs $P(\Delta u)$ and $P(l)$ of all three cases differ wildly (not shown) and that they are only similar when normalised with $l_{m}$ and $\sigma$, respectively.

\subsection{Conditional means}

Finally, we consider the conditional means $\left\langle[\Delta u]^{n} \mid l\right\rangle$ for $n=1 \ldots 3$, i.e. the mean velocity difference conditioned on the segment length. This is similar to structure functions $D_{n}=\left\langle\left[\Delta u_{1}\right]^{n}\left(r_{i}\right)\right\rangle=\left\langle\left[u_{1}\left(x_{i}+r_{i}\right)-u_{1}\left(x_{i}\right)\right]^{n}\right\rangle$ which are the velocity difference of two points separated by the vector $r_{i}$ and were introduced by Kolmogorov ((Kolmogorov 1941b, Kolmogorov 1941a)).

When evaluating structure functions, the separation vector $r_{i}$ is fixed in space, independent the local flow topology. This is obviously not the case for $\left\langle[\Delta u]^{n} \mid l\right\rangle$, where the separation is equal to the segment length and orientated along the streamline. We may thus expect that $\left\langle[\Delta u]^{n} \mid l\right\rangle$ takes the local flow into account and that the velocity along the streamline is more correlated than along the arbitrary separation vector $r_{i}$. However, we must keep in mind that the separation vector $r_{i}$ is arbitrarily large whereas the streamline length $l$ has a maximum value which is about $5 l_{m}$ where $l_{m}$ is the mean length of streamline segments. Schaefer et al. (2012b) have shown that $l_{m}=(\eta \lambda)^{1 / 2}$, i.e. that $l_{m}$ is the geometric mean of the Kolmogorov length $\eta$ and the Taylor length $\lambda$ (Schaefer et al. 2012b). Hence we should not expect the range of $l$ to be comparable to an inertial range. The streamline segment statistics mostly explore the dissipation range.

The first moment $\langle\Delta u \mid l\rangle$ is shown in both figure 6(a) and figure 6(b), Note that, unlike structure functions, the first moment does not vanish even in statistically stationary periodic turbulence due to the characteristic differences between positive and negative segments as highlighted by the asymmetry of the jpdfs. For statistically stationary periodic turbulence and near-field grid-generated decaying turbulence, we find that for very small segments $l / l_{m} \lesssim 0.5,\langle\Delta u \mid l\rangle \sim 0$. Intermediate segments $0.5 \lesssim l / l_{m} \lesssim 2$ have a negative mean velocity difference, while $\langle\Delta u \mid l\rangle>0$ for large segments $2 \lesssim l / l_{m}$. In agreement with the findings of Wang (2009) and Wang \& Peters (2010), we find a linear relation of the form $\langle\Delta u \mid l\rangle=\alpha_{\infty} l / l_{m}$ for large $l / l_{m}$. Wang (2009) showed that the velocity difference along scalar trajectories (i.e. dissipation elements) does scale linearly with $l$ when $l$ is large. As dissipation elements and streamline segments are conceptually related inasmuch as that they depend on the flow structure, the linear increase as seen in figure 6(a) is not completely surprising, although the theory 


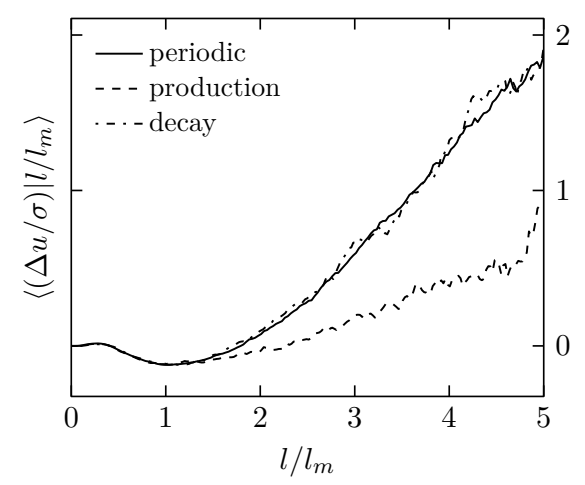

(a)

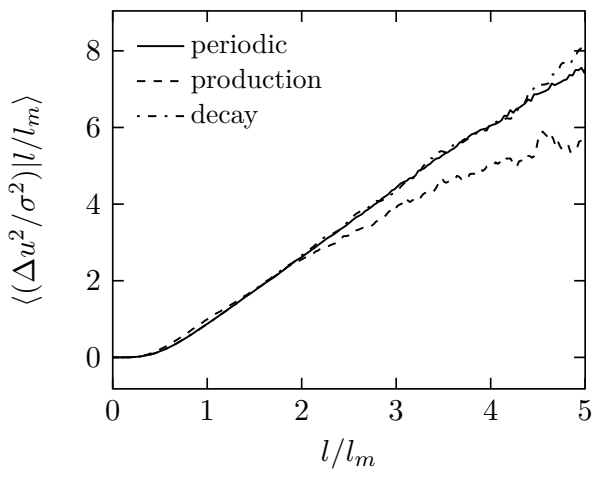

(c)

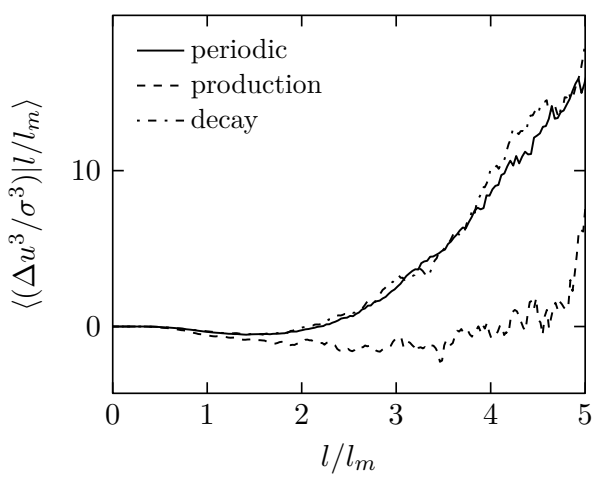

(e)

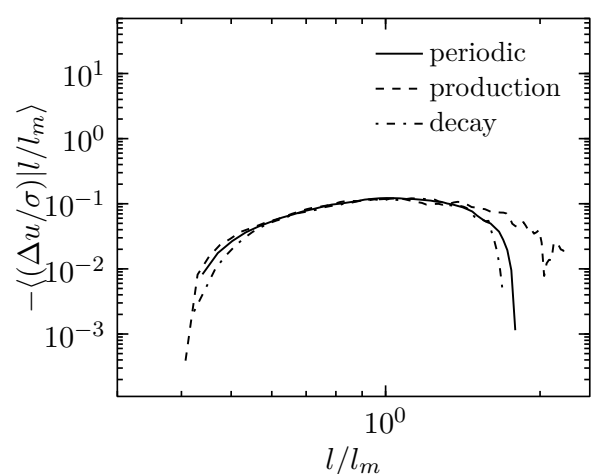

(b)

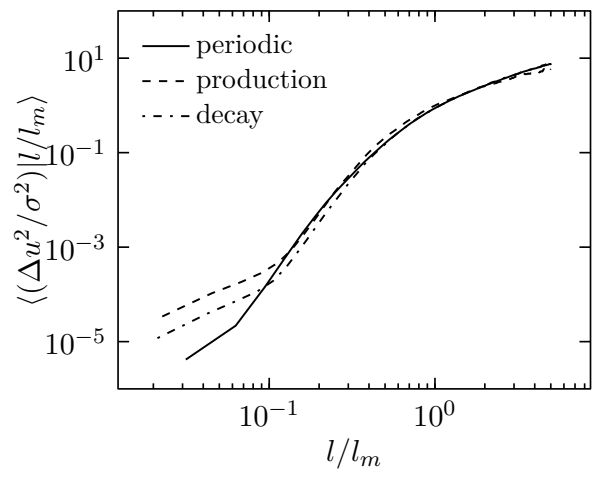

(d)

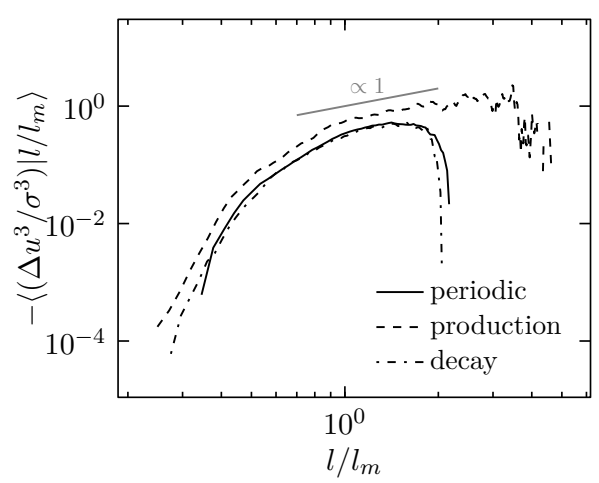

(f)

Figure 6. Conditional means of $\left\langle[\Delta u]^{n} \mid l\right\rangle$ normalised with $\sigma$ and the mean length $l_{m}$. (a) and (b) $n=1$, (c) and (d) $n=2$, (e) and (f) $n=3$.

can not be carried over straightforwardly. Thus, figure 6(a) implies that intermediate segments are compressed while large segments are stretched, in agreement with the jpdfs figure 4(c) above. In fact, there is very good agreement between the isotropic data and the downstream fractal flow. The production region data show qualitatively similar behaviour, but with a wider range of segment lengths with negative mean velocity difference and a smaller slope for large segments. This might indicate that it could be possible to collapse all three plots at large $l$ if normalised with suitable quantities, as 
Gampert, Goebbert, Schaefer, Gauding, Peters, Aldudak \& Oberlack (2011) were able to collapse $\langle\Delta u \mid l\rangle$ along dissipation elements for five different flow types (shear flow, forced turbulence, decaying turbulence, channel flow and Kolmogorov flow) using the timescale $\tau=k / \varepsilon$ and the strain rate $\alpha_{\infty}$. Figure 6(b) highlights the region for which $\langle\Delta u \mid l\rangle<0$. We find a remarkably good agreement between all our data for intermediate segment lengths.

The second moment $\left\langle[\Delta u]^{2} \mid l\right\rangle$ is shown in figure 6(c) and figure 6(d). Obviously, $\left\langle[\Delta u]^{2} \mid l\right\rangle \geq 0$ for all $l$. For small $l$, one should not necessarily expect a $l^{2}$ scaling of $\left\langle[\Delta u]^{2} \mid l\right\rangle$ as $l$ is also a random variable and one cannot apply a Taylor expansion argument in the same way that one can apply it on structure functions $\left\langle\left[\Delta u_{1}\right]^{2}\right\rangle \sim$ $\left\langle\left[\Delta u_{2}\right]^{2}\right\rangle \sim\left\langle\left[\Delta u_{3}\right]^{2}\right\rangle \sim r^{2}$ for $r \rightarrow 0$. Hence, $\left\langle[\Delta u]^{2} \mid l\right\rangle$ does not necessarily scale as $l^{2}$ for $l \rightarrow 0$. In fact we find for $l \rightarrow 0$ a larger scaling exponent for all datasets.

For large $l$, we find a linear increase of $\left\langle[\Delta u]^{2} \mid l\right\rangle$ with $l$. Similarly to the first moment, the statistically stationary periodic turbulence data and the grid-generated turbulence decay data agree very well, while the production region data close to the grid exhibits a different slope for large segments. Curiously, for small $l / l_{m}$, the periodic turbulence data agrees better with the production region data than with the decay region data. However, for $l / l_{m} \gtrsim 0.5$, the periodic and the decay data agree very well, cf. figure 6(d). We note that $\left\langle[\Delta u]^{2} l\right\rangle$ starts with a scaling larger than $l^{2}$ and then scales linearly with $l$ for large segments.

The third moment $\left\langle[\Delta u]^{3} \mid l\right\rangle$ is presented in figure 6(e) and figure 6(f). As expected, we find a similar behaviour as in case of the first moment. However, the range of $l / l_{m}$ for which $\left\langle[\Delta u]^{3} \mid l\right\rangle<0$ is larger than the corresponding range for the first moment. This agrees with the jpdfs inasmuch as large values of $\Delta u$ for a certain $l$ are now higher weighted and that $|\Delta u|$ was found to be higher for negative segments than for positive segments. Again, we find that the statistically stationary periodic turbulence and the grid-generated decaying turbulence data agree very well and collapse for all $l / l_{m}$, while the production region data shows qualitatively similar behaviour. The region $\left\langle[\Delta u]^{3} \mid l\right\rangle<0$ is of particular interest, as the asymmetry in the joint PDF of Figure 4 is related to the turbulence cascade process (see end of section 4.1) and one might ask whether the negative sign of $\left\langle[\Delta u]^{3} \mid l\right\rangle$ reflects the forward nature of this process at dissipative scales in the same way that the negative sign in Kolmogorov's $\left\langle\left[\Delta u_{1}\right]^{3}\right\rangle=-4 / 5\langle\varepsilon\rangle r$ reflects the forward nature of the cascade process in inertial scales. We cannot answer this question in this paper but we do investigate the scaling with $r$ of $-\left\langle[\Delta u]^{3} \mid l\right\rangle$ in figure $6(\mathrm{f})$. There is no such power-law scaling range for the periodic and the decaying turbulence data, but there may be a very short one with exponent 1 for the highly non-gaussian data in the production region of the grid-generated turbulence exhibits.

This observation is consonant with the finding of Laizet, Vassilicos \& Cambon (2013), Gomes-Fernandes et al. (2015) and Laizet, Nedić \& Vassilicos (2015b) that the best $-5 / 3$ power law spectrum over the entire grid-generated turbulence is found in the production region. It may be that the non-gaussianity in the production region has 
the same cause as these well-defined power laws, namely the sharp interfaces between alternating potential and vortical flow patches. The cause of these well-defined power laws has nothing to do with Kolmogorov's theory as already noted by Laizet et al. (2013), Gomes-Fernandes et al. (2015) and Laizet et al. (2015b).

It could be possible that this very small scaling range (if it really is one) in the production region would slowly increase with Reynolds number. This would imply that the jpdfs in figure 4 are not universal and depend on the Reynolds number as well. Examining this dependency would therefore be a good starting point for further studies of $\left\langle[\Delta u]^{n} \mid l\right\rangle$.

\section{Conclusion}

The asymmetry in Figure 4 which shows that there are longer streamline segments with positive than with negative velocity difference $\Delta u$ is a dynamic effect reflecting inertial cascade processes at dissipative scales. The turbulence cascade mechanism is expressed in terms of structure functions and their power-law dependence on twopoint separation distance in the so-called inertial range of length-scales. The statistical quantities $\left\langle[\Delta u]^{n} \mid l\right\rangle$ studied in this paper are defined over a range of streamline segment lengths which extends up to no more than an order of magnitude higher than $l_{m}=\sqrt{\eta \lambda}$. Hence, the range of scales sampled by the streamline segments statistics $\left\langle[\Delta u]^{n} \mid l\right\rangle$ is mostly dissipative. Nevertheless, the asymmetry in the jpdf underlying these statistics is an asymmetry between strain and compression and can only reflect the time-irreversible energy cascade from large to small scales. Hence, the streamline segment statistics $\left\langle[\Delta u]^{n} \mid l\right\rangle$ are capable of picking up cascade information but at the dissipation range level. They are also capable of displaying approximate linear dependencies on $l$ if $l$ is not much smaller than one or two $l_{m}$ depending on case.

The comparison we have made between our three DNS data sets suggests that, even if sensitive to the average direction of the cascade, streamline statistics are not very sensitive to the difference between equilibrium and non-equilibrium cascades. Indeed, the grid-generated decaying turbulence and the statistically stationary periodic turbulence have very similar such statistics. However, these statistics do seem to be sensitive to the difference between gaussian and non-gaussian turbulence given that the turbulence in the production region of our grid-generated flow is highly non-gaussian (see Mazellier \& Vassilicos (2010) and Laizet et al. (2015b)) whereas the turbulence in the other two DNS data sets is gaussian.

\section{Acknowledgment}

Jonas Boschung and Norbert Peters were supported by the Deutsche Forschungsgemeinschaft under grant Pe241/38-2 and gratefully acknowledge the computing time granted by the JARA-HPC-Vergabegremium provided on the JARA-HPC Partition part of the supercomputer JUQUEEN at the Forschungszentrum Jülich. Sylvain Laizet and Chris- 
tos Vassilicos acknowledge the UK turbulence Consortium (EPSRC Research grant EP/L000261/1) for access to UK supercomputing resources and PRACE for awarding us access to resource SUPERMUC based in Germany at Leibniz-Rechenzentrum (Leibniz Supercomputing Centre). They were supported by an ERC Advanced Grant (2013-2018) awarded to J.C.Vassilicos.

\section{References}

Eswaran, V \& SB Pope (1988), 'Direct numerical simulations of the turbulent mixing of a passive scalar', Phys. Fluids 31, 506-520.

Gampert, Markus, Jens Henrik Goebbert, Philip Schaefer, Michael Gauding, Norbert Peters, Fettah Aldudak \& Martin Oberlack (2011), 'Extensive strain along gradient trajectories in the turbulent kinetic energy field', New Journal of Physics 13(4), 043012.

Gomes-Fernandes, R., B. Ganapathisubramani \& J.C. Vassilicos (2015), 'The energy cascade in nearfield non-homogeneous non-isotropic turbulence', J. Fluid Mech. 771, 676-705.

Goto, S. \& J. C. Vassilicos (2015), 'Energy dissipation and flux laws for unsteady turbulence without Richardson-Kolmogorov cascade', Phys. Letter A 379(16-17), 1144-1148.

Hou, Thomas Y \& Ruo Li (2007), 'Computing nearly singular solutions using pseudo-spectral methods', J. Comp. Phys. 226(1), 379-397.

Hurst, D. \& J. C. Vassilicos (2007), 'Scalings and decay of fractal-generated turbulence', Phys. Fluids 19(035103).

Kolmogorov, A.N. (1941a), 'Dissipation of energy in locally isotropic turbulence', Dolk. Akad. Nauk SSSR 32, 19-21.

Kolmogorov, A.N. (1941b), 'The local structure of turbulence in incompressible viscous fluid for very large reynolds numbers', Dolk. Akad. Nauk SSSR 30, 299-303.

Laizet, S. \& E. Lamballais (2009), 'High-order compact schemes for incompressible flows: a simple and efficient method with the quasi-spectral accuracy', J. Comp. Phys. 228(16), 5989-6015.

Laizet, S., J. Nedić \& J.C. Vassilicos (2015a), 'Influence of the spatial resolution on fine-scale features in dns of turbulence generated by a single square grid', Int. J. of Comp. Fluid Dynamics 29(35), 286-302.

Laizet, S., J.C. Vassilicos \& C. Cambon (2013), 'Interscale energy transfer in decaying turbulence and vorticity-strain-rate dynamics in grid-generated turbulence', Fluid Dynamics Research 45(6), 061408.

Laizet, S. \& N. Li (2011), 'Incompact3d, a powerful tool to tackle turbulence problems with up to $\mathrm{O}\left(10^{5}\right)$ computational cores', Int. J. Numer. Methods Fluids 67(11), 1735-1757.

Laizet, Sylvain, J Nedić \& J Christos Vassilicos (2015b), 'The spatial origin of- 5/3 spectra in gridgenerated turbulence', Physics of Fluids (1994-present) 27(6), 065115.

Lele, S. K. (1992), 'Compact finite difference schemes with spectral-like resolution', J. Comp. Phys. 103, $16-42$.

Li, N. \& S. Laizet (2010), 2DECOMP \& FFT-A highly scalable 2D decomposition library and FFT interface, in 'Cray User Group 2010 conference', pp. 1-13.

Mazellier, N. \& J. C. Vassilicos (2010), 'Turbulence without Richardson-Kolmogorov cascade', Phys. Fluids 22, 075101.

Schaefer, P., M. Gampert \& N. Peters (2012a), 'The length distribution of streamline segments in homogeneous isotropic decaying turbulence', Phys. Fluids 24, 045104.

Schaefer, P, M Gampert \& N Peters (2013), 'A model equation for the joint distribution of the length and velocity difference of streamline segments in turbulent flows', Phys. Fluids 25(11), 115107.

Schaefer, Philip, Markus Gampert \& Norbert Peters (2012b), 'On the scaling of the mean length of streamline segments in various turbulent flows', Comptes Rendus Mécanique 340(11-12), 859866 . 
Simmons, L.F.G. \& C. Salter (1934), 'Experimental investigation and analysis of the velocity variations in turbulent flow', Proc. Roy. Soc. A 145 (854), 212-234.

Vassilicos, J.C. (2015), 'Dissipation in turbulent flows', Ann. Rev. Fluid Mech. 47, 95-114.

Wang, L. (2009), 'Scaling of the two-point velocity difference along scalar gradient trajectories in fluid turbulence', Physical Review E 79(4), 046325.

Wang, L. (2010), 'On properties of fluid turbulence along streamlines', J. Fluid Mech. 648, 183-203.

Wang, L. \& N. Peters (2010), 'Mean velocity increment conditioned on gradient trajectories of various scalar variables in turbulence', Phys. Scripta 2010(T142), 014004. 